\title{
Respostas Fisiológicas à Caminhada Máxima e Submáxima em Pacientes com Doença Arterial Periférica Sintomática
}

\author{
Physiological Responses to Maximal and Submaximal Walking in Patients with Symptomatic Peripheral \\ Artery Disease
}

Marcel Chehuen, ${ }^{\circledR}$ Aluisio Andrade-Lima, ${ }^{2}$ Natan Silva Junior, ${ }^{1}$ Roberto Miyasato, ${ }^{1}$ Rodrigo W. Alves de Souza, ${ }^{1}$ Anthony Leicht, ${ }^{3}$ Patricia Chakur Brum, ${ }^{1}$ Edilamar M. Oliveira, ${ }^{10}$ Nelson Wolosker, ${ }^{4}{ }^{\bullet}$ Claudia Lucia de Moraes

Forjaz ${ }^{10}$

Universidade de São Paulo - Escola de Educação Física e Esportes, ${ }^{1}$ São Paulo, SP - Brasil

Universidade Federal de Sergipe, ${ }^{2}$ Aracaju, SE - Brasil

James Cook University, ${ }^{3}$ Townsville, Queensland - Austrália

Hospital Israelita Albert Einstein, ${ }^{4}$ São Paulo, SP - Brasil

\section{Resumo}

Fundamento: Embora a caminhada máxima e submáxima sejam recomendadas para pacientes com doença arterial periférica (DAP), a realização desses exercícios pode induzir diferentes respostas fisiológicas.

Objetivos: Comparar os efeitos agudos de caminhada máxima e submáxima na função cardiovascular, a regulação e os processos fisiopatológicos associados pós-exercício em pacientes com DAP sintomática.

Métodos: Trinta pacientes do sexo masculino foram submetidos a 2 sessões: caminhada máxima (protocolo de Gardner) e caminhada submáxima (15 períodos de 2 minutos de caminhada separados por 2 minutos de repouso ereto). Em cada sessão, foram medidos a pressão arterial (PA), a frequência cardíaca (FC), a modulação autonômica cardíaca (variabilidade da FC), os fluxos sanguíneos (FS) do antebraço e da panturrilha, a capacidade vasodilatadora (hiperemia reativa), o óxido nítrico $(\mathrm{ON})$, o estresse oxidativo (a peroxidação lipídica) e a inflamação (quatro marcadores), pré e pós-caminhada. ANOVAs foram empregadas e p $<0,05$ foi considerado significativo.

Resultados: A PA sistólica e a PA média diminuíram após a sessão submáxima, mas aumentaram após a sessão máxima (interações, $\mathrm{p}<0,001$ para ambas). A PA diastólica não foi alterada após a sessão submáxima $(\mathrm{p}>0,05)$, mas aumentou após a caminhada máxima (interação, $p<0,001$ ). A FC, o equilíbrio simpatovagal e os $\mathrm{FS}$ aumentaram de forma semelhante após as duas sessões (momento, $p<0,001, p=0,04$ e $p<0,001$, respectivamente), enquanto a capacidade vasodilatadora, o $\mathrm{ON}$ e o estresse oxidativo permaneceram inalterados $(p>0,05)$. As moléculas de adesão vascular e intercelular aumentaram de forma semelhante após as sessões de caminhada máxima e submáxima (momento, $\mathbf{p}=\mathbf{0 , 0 0 1}$ ).

Conclusões: Nos pacientes com a DAP sintomática, a caminhada submáxima, mas não a máxima, reduziu a PA pósexercício, enquanto a caminhada máxima manteve a sobrecarga cardíaca elevada durante o período de recuperação. Por outro lado, as sessões de caminhada máxima e submáxima aumentaram a FC, o equilíbrio simpatovagal cardíaco e a inflamação pós-exercício de forma semelhante, enquanto não alteraram a biodisponibilidade de ON e o estresse oxidativo pós-exercício.

Palavras-chave: Caminhada; Doença Arterial Periférica; Velocidade da Caminhada; Monitoração Hemodinâmica; Claudicação Intermitente; estresse Oxidativo; Biomarcadores.

\section{Abstract}

Background: Although maximal and submaximal walking are recommended for patients with peripheral artery disease (PAD), performing these exercises may induce different physiological responses.

Objectives: To compare the acute effects of maximal and submaximal walking on post-exercise cardiovascular function, regulation, and associated pathophysiological processes in patients with symptomatic PAD.

Correspondência: Aluisio Andrade-Lima •

Universidade Federal de Sergipe - Rua João Geniton da Costa, 306. CEP 49095-796, Aracaju, SE - Brasil

E-mail: aluisiolima@live.com

Artigo recebido em 27/02/2020, revisado em 25/06/2020, aceito em 12/08/2020

DOI: https://doi.org/10.36660/abc.20200156 
Methods: Thirty male patients underwent 2 sessions: maximal walking (Gardner's protocol) and submaximal walking (15 bouts of 2 minutes of walking separated by 2 minutes of upright rest). In each session, blood pressure (BP), heart rate (HR), cardiac autonomic modulation (HR variability), forearm and calf blood flows (BF), vasodilatory capacity (reactive hyperemia), nitric oxide (NO), oxidative stress (lipid peroxidation), and inflammation (four markers) were measured pre- and post-walking. ANOVAs were employed, and p < 0.05 was considered significant.

Results: Systolic and mean BP decreased after the submaximal session, but they increased after the maximal session (interactions, $p$ < 0.001 for both). Diastolic BP did not change after the submaximal session ( $p>0.05$ ), and it increased after maximal walking (interaction, $p<0.001$ ). HR, sympathovagal balance, and BF increased similarly after both sessions (moment, $p<0.001, p=0.04$, and $p<0.001$, respectively), while vasodilatory capacity, NO, and oxidative stress remained unchanged ( $p>0.05$ ). Vascular and intercellular adhesion molecules increased similarly after both maximal and submaximal walking sessions (moment, $p=0.001$ ).

Conclusions: In patients with symptomatic PAD, submaximal, but not maximal walking reduced post-exercise BP, while maximal walking maintained elevated cardiac overload during the recovery period. On the other hand, maximal and submaximal walking sessions similarly increased post-exercise HR, cardiac sympathovagal balance, and inflammation, while they did not change post-exercise NO bioavailability and oxidative stress.

Keywords: Walking; Peripheral Arterial Disease; Walking Speed; Hemodynamic Monitoring; Intermittent Claudication; Oxidative Stress; Byomarkers.

Full texts in English - http://www.arquivosonline.com.br

\section{Introdução}

A doença arterial periférica (DAP) caracteriza-se pelo estreitamento das artérias dos membros inferiores, convencionalmente devido à aterosclerose. ${ }^{1,2}\{$ Norgren, 2007, Inter-society consensus for the management of peripheral arterial disease Pacientes no segundo estágio da doença (pela classificação de Fontaine) apresentam um sintoma conhecido como claudicação intermitente $(\mathrm{Cl})$, que é caracterizada pelo aparecimento de dor na parte inferior da perna durante a caminhada que é aliviada com repouso. ${ }^{1,2}$ Além disso, pacientes com a DAP sintomática podem apresentar valores elevados de pressão arterial (PA), ${ }^{3}$ sobrecarga cardiovascular, ${ }^{3,4}$ disfunção autonômica cardíaca, ${ }^{4}$ disfunção endotelial, estresse oxidativo exacerbado e inflamação. ${ }^{5-7}$ Todas estas manifestações fisiológicas contribuem para a progressão da doença e a morbimortalidade cardiovascular. ${ }^{2,3,6}$

O treinamento de exercício tem sido considerado o melhor tratamento para pacientes com a Cl. ${ }^{1,2} \mathrm{O}$ treinamento regular melhora a capacidade de locomoção, os sintomas de claudicação, a qualidade de vida e a saúde cardiovascular desses pacientes. ${ }^{1,8,9}$ Entre as diversas modalidades de treinamento, a caminhada tem sido amplamente recomendada por várias diretrizes. ${ }^{1,2,9}$ No entanto, acreditase que os efeitos crônicos do treinamento resultem da soma das respostas agudas às sessões, ${ }^{10}$ o que reforça a importância da realização de sessões diárias de caminhada para otimizar as adaptações crônicas. Porém, agudamente, cada sessão de caminhada pode transitoriamente aumentar o risco cardiovascular. ${ }^{10}$ De fato, estudos prévios têm relatado que caminhar até sintomas próximos ao máximo da $\mathrm{Cl}$ aumenta a sobrecarga cardíaca, disfunção endotelial, estresse oxidativo e inflamação, ${ }^{7,11-13}$ o que aumenta o risco de isquemia e arritmias em pacientes predispostos. ${ }^{14}$

Nesse sentido, a caminhada máxima pode ter efeitos perigosos pós-exercício em pacientes com a DAP sintomática, e a caminhada submáxima (até dor moderada nas pernas) se apresenta como uma opção potencial para promover menor sobrecarga cardíaca pós-exercício, acompanhada por estresse oxidativo e inflamação moderados. Novakovic et al., ${ }^{15}$ mostraram que caminhar com dor moderada melhorou vários desfechos nesses pacientes, como a função vascular. Adicionalmente, estudos anteriores testaram um protocolo específico de caminhada submáxima (15 períodos de 2 minutos de caminhada no limiar da dor), relatando que induziu níveis toleráveis de dor nas pernas, bem como estímulos metabólicos e cardiovasculares moderados durante a sua execução, ${ }^{16}$ induziu hipotensão pós-exercício, ${ }^{17}$ e melhorou a capacidade de caminhada e parâmetros cardiovasculares após um período de treinamento regular. ${ }^{8}$

Desta maneira, o objetivo do presente estudo foi comparar, em pacientes com DAP sintomática, os efeitos agudos de exercícios máximos e submáximos de caminhada nas seguintes variáveis pós-exercício: i) a função cardiovascular, avaliada pela PA, a frequência cardíaca (FC) e o duplo-produto (DP); ii) a modulação autonômica cardíaca, avaliada pelos componentes da variabilidade da FC de frequência baixa (FB) e alta (FA) e pela relação $F B / F A$; iii) a função vascular, avaliada pelos fluxos sanguíneos (FS) do antebraço e da panturrilha e pelas respostas dos FS à hiperemia reativa; iv) a função endotelial, avaliada pela biodisponibilidade de óxido nítrico $(\mathrm{ON})$; v) o estresse oxidativo, avaliado pela peroxidação lipídica; e vi) a inflamação, avaliada pela proteína C-reativa (CRP), o fator de necrose tumoral- $\alpha$ (TNF- $\alpha$ ), a molécula de adesão de células vasculares (VCAM) e a molécula de adesão intercelular (ICAM). As hipóteses eram as seguintes: i) a sessão máxima de caminhada aumentaria a sobrecarga cardíaca pósexercício (PA, FC e DP), o equilíbrio simpatovagal (FB e relação FB/FA) e a disfunção vascular, enquanto a sessão de caminhada submáxima diminuiria a PA e o DP, enquanto induziria um aumento menor da FC e do equilíbrio simpatovagal; e ii) as sessões máximas e submáximas de caminhada aumentariam o estresse oxidativo pós-exercício e a inflamação com maiores respostas após a caminhada máxima.

\section{Métodos}

Este estudo unicêntrico seguiu um desenho de medição repetida não aleatória em que cada paciente foi submetido a duas sessões experimentais conduzidas em uma ordem fixa. O protocolo do estudo estava de acordo com a Declaração de Helsinque. Foi registrado no site Brasileiro 
de Ensaios Clínicos (http://www.ensaiosclinicos.gov.br, RBR-3pq58k) e aprovado pelo Comitê Conjunto de Ética em Pesquisa em Seres Humanos da Escola de Educação Física e Esporte da Universidade de São Paulo (processo 667.382). Foi obtido o consentimento informado por escrito de todos os participantes.

\section{Participantes}

Os pacientes foram recrutados entre os atendidos na Unidade Vascular do Hospital das Clínicas da Universidade de São Paulo, Brasil, de acordo com a possibilidade de entrar em contato com eles. Foram convidados pacientes do sexo masculino com diagnóstico prévio de DAP e Cl. Os critérios de inclusão foram os seguintes: a) idade $\geq 50$ anos; b) índice tornozelo-braquial $\leq 0,90$ em pelo menos uma perna ${ }^{1}$; c) estágio II (a e b) de DAP pela classificação de Fontaine ${ }^{1}$; d) índice de massa corporal $<\mathrm{kg} / \mathrm{m}^{2}$; e) PA sistólica de repouso $<160$ mmHg e PA diastólica de repouso < 105 mmHg; f) não uso de $\beta$-bloqueadores ou bloqueadores dos canais de cálcio não diidropiridínicos; g) a capacidade de caminhar durante pelo menos 2 minutos a 3,2 km/h em esteira; h) a capacidade de realizar um teste incremental em esteira limitada por sintomas da $\mathrm{Cl}$; e i) a ausência de isquemia miocárdica ou arritmias complexas durante teste em esteira.

\section{Avaliações preliminares}

Todos os pacientes foram submetidos a uma avaliação preliminar para identificar se atendiam aos critérios do estudo. Foram entrevistados para avaliar o seguinte: idade, presença de doença cardiovascular, fatores de risco, comorbidades e medicação atual. O índice tornozelo-braquial foi medido conforme descrito anteriormente. ${ }^{1}$ A massa corporal e a estatura foram avaliadas com equipamento padrão (Welmy 110, Brasil) e foi calculado o índice de massa corporal. Foi medida a PA braquial em repouso pelo método auscultatório após 5 minutos de repouso sentado. Três medições foram realizadas em cada uma das 2 visitas e foi calculado o valor médio para cada braço. Também foi documentado o maior valor médio. Por fim, todos os pacientes realizaram um teste ergométrico em esteira seguindo o protocolo de Gardner (3,2 $\mathrm{km} / \mathrm{h}$ com aumento de $2 \%$ no grau por minuto $)^{18}$ até que ocorresse dor máxima de claudicação. Este teste também foi empregado como uma familiarização ao esforço máximo.

\section{Protocolo experimental}

Após os procedimentos preliminares, os pacientes que preencheram todos os critérios do estudo foram submetidos ao protocolo experimental que consistia em ambas as sessões experimentais, caminhada máxima e submáxima. A sessão de caminhada submáxima foi realizada após a sessão máxima com intervalo de pelo menos 7 dias entre as sessões. Além disso, todos os pacientes realizaram 2 sessões de familiarização antes de serem submetidos à sessão submáxima. Para cada sessão, foram avaliadas as variáveis cardiovasculares, autonômicas, endoteliais, de estresse oxidativo e inflamatórias antes e depois dos protocolos de caminhada submáxima ou máxima.

Antes de ambas as sessões, os pacientes foram orientados a manter rotinas semelhantes durante as 24 horas anteriores.
Adicionalmente, foram orientados a evitar exercício físico durantes as 48 horas anteriores, bebidas alcoólicas durante as 24 horas anteriores e uso de tabaco no dia das sessões. Também foram orientados a tomar a medicação regularmente e a comparecer ao laboratório em estado de jejum.

As sessões foram realizadas em laboratório com temperatura controlada $\left(20\right.$ a $\left.22^{\circ} \mathrm{C}\right)$. Os pacientes chegaram às 7 horas e receberam uma refeição padronizada ( 2 barras de cereais e $50 \mathrm{ml}$ de suco). ${ }^{19,20}$ Em seguida, um cateter foi inserido na veia antecubital do braço esquerdo e mantido patente por solução salina estéril. Os pacientes então permaneceram em posição supina durante 20 minutos até o início dos procedimentos experimentais.

Foram iniciados os procedimentos experimentais às 8 horas com avaliações pré-exercício realizadas na posição supina após um período de estabilização de 10 minutos. Eletrocardiograma (ECG) e respiração foram registrados entre 10 e 20 minutos para avaliar a modulação autonômica cardíaca. Foram medidas a PA e a FC auscultatórias em triplicata entre 20 e 25 minutos e foi utilizado o valor médio para análise. Subsequentemente, foi coletada uma amostra de sangue venoso, seguida da avaliação dos FS dos membros inferiores e superiores e das respostas vasodilatadoras à hiperemia reativa.

Na sequência, os pacientes realizaram o exercício de caminhada em esteira. Na sessão máxima, caminharam a $3,2 \mathrm{~km} / \mathrm{h}$ com o aumento de grau de $2 \%$ a cada minuto até a dor máxima (protocolo de Gardner). ${ }^{18}$ Durante a sessão submáxima, realizaram 15 períodos de 2 minutos de caminhada separados por 2 minutos de repouso ereto, conforme descrito anteriormente. ${ }^{8,16,17}$ A velocidade da esteira foi mantida em 3,2 km/h com o grau ajustado para manter a FC do limiar de dor (ou seja, a FC medida quando os pacientes experimentaram dor claudicante inicial durante o teste de caminhada máxima preliminar).

Ao final das sessões de caminhada, os pacientes retornaram imediatamente à posição supina para as avaliações pósexercício que incluíram uma amostra imediata de sangue. Dos 20 aos 30 minutos de recuperação, foram registrados o ECG e os movimentos respiratórios para avaliação da modulação autonômica cardíaca, seguida pelas avaliações da PA e FC auscultatórias em triplicata. Finalmente, foram registrados os FS e as respostas vasodilatadoras.

\section{Medidas}

\section{Função cardiovascular}

Foram obtidos os registros do ECG no D2 (EMG System, Brasil) com a FC determinada pelo ECG. Foi obtido o sinal respiratório por uma cinta piezoelétrica (UFI, Pneumotrace2, EUA) posicionado no tórax dos pacientes. Foi medida a PA auscultatória no braço dominante utilizando um esfigmomanômetro de mercúrio (Unitec, Brasil), e foi calculada a PA média. O DP foi calculado pelo produto da FC e da PA sistólica como um marcador do consumo de oxigênio miocárdico e, portanto, da sobrecarga cardíaca. ${ }^{21}$ 


\section{Modulação autonômica cardíaca}

Para a avaliação autonômica cardíaca, os intervalos R-R do ECG e os sinais respiratórios da cinta torácica foram inseridos em um sistema de aquisição de dados (WinDaq, DI-720, Akron, EUA), com uma taxa de amostragem de $500 \mathrm{~Hz} /$ canal. Foram analisados segmentos estacionários de 250 a 300 batimentos por meio de análise espectral da variabilidade da FC usando o método autoregressivo (Heart Scope, versão 1.3.0.1, AMPS-LLC, EUA). Os componentes da variabilidade da $F C$ de $F B\left(F_{R R^{\prime}} 0,04-0,15 \mathrm{~Hz}\right)$ e $F A$ $\left(F A_{R R^{\prime}} 0,15-0,4 \mathrm{~Hz}\right)$ foram calculados e expressos em unidades normalizadas (un). Também foi calculada a relação FB/FA. Todos os procedimentos seguiram a Task Force for HR variability. ${ }^{22}$

\section{Função vascular}

Foram determinados os FS simultaneamente no antebraço dominante e na perna com o menor índice tornozelo-braquial, via pletismografia de oclusão venosa (Hokanson, AI6, EUA). ${ }^{23}$ Resumidamente, os FS da mão e do pé foram interrompidos por manguitos insuflados a 200 $\mathrm{mmHg}$ posicionados, respectivamente, ao redor do punho e tornozelo. Outros manguitos colocados no braço e na coxa foram insuflados rapidamente por 10 segundos a 40 a $60 \mathrm{mmHg}$, seguidos por 10 segundos de desinsuflação. Foram detectados aumentos nos volumes do antebraço e da panturrilha por medidores de pressão de mercúrio, posicionados na maior circunferência desses segmentos dos membros e registrados por software especializado (NIVP3; Hokanson, EUA). Foram realizadas as medições durante 4 minutos (12 ciclos de 20 segundos) e as primeiras 2 e a última medição do ciclo foram excluídas da análise (ou seja, uma média de 9 ciclos). As respostas vasodilatadoras do antebraço e da panturrilha à hiperemia reativa foram avaliadas imediatamente após a determinação dos FS. ${ }^{23}$ Para isso, foi ocluído o FS para cada membro durante 5 minutos com a insuflação dos manguitos da coxa e do antebraço a $200 \mathrm{mmHg}$. Em seguida, os manguitos foram desinsuflados e foram medidos os FS pós-oclusão durante 4 minutos, conforme descrito anteriormente. A resposta vasodilatadora foi calculada como a diferença na área sob a curva (AUCFS) das medições de FS pós e pré-hiperemia

\section{Análise de sangue}

Em cada momento de amostragem, foram coletados $15 \mathrm{ml}$ de sangue em tubos vacutainer tratados com EDTA anticoagulante de padrão. As amostras foram centrifugadas em até 30 minutos, divididas em alíquotas e armazenadas $\mathrm{a}-80{ }^{\circ} \mathrm{C}$ até a análise. Foram determinadas as concentrações plasmáticas de CRP, TNF- $\alpha$, VCAM e ICAM por ensaios imunoenzimáticos (ELISA) de acordo com as instruções do fabricante de cada kit (Cayman Chemical, EUA para CRP; and R\&D Systems, EUA para TNF- $\alpha$, VCAM e ICAM). A peroxidação lipídica foi analisada por kits específicos (Cayman Chemical, EUA), e o ON foi analisado pelo método de quimioluminescência com um analisador específico (Sievers ${ }^{\circledR}$ Nitric Oxide Analyzer ONA 280, EUA).

\section{Análises estatísticas}

Considerando um poder de $90 \%$, um erro alfa de $5 \%$ e um desvio padrão de $3 \mathrm{mmHg}$ para a PA sistólica e 0,6 ml.100 $\mathrm{ml}$ tecido $^{-1} \cdot \mathrm{min}^{-1}$ a para BF (ou seja, os desfechos clínicos principais), os tamanhos mínimos de amostra necessários para detectar uma diferença de $4 \mathrm{mmHg}$ na PA sistólica e de $0,5 \mathrm{ml} .100 \mathrm{ml}$ tecido-1 $\mathrm{min}^{-1}$ na BF foram calculados em 10 e 14 indivíduos, respectivamente. Tendo em vista que foram incluídas outras variáveis com maior variação no estudo, o tamanho de amostra utilizado foi maior.

Foram verificadas a normalidade e a homogeneidade de variância para todos os dados pelos testes de Shapiro-Wilk e Levene, respectivamente. Quando identificada a não normalidade dos dados, foi aplicada uma transformação logarítmica e foi obtida a distribuição normal. As respostas às sessões de caminhada foram comparadas por ANOVA bidirecional (Statsoft, Statistic for Windows 4.3, Oklahoma, EUA) para medidas repetidas com a sessão (máxima versus submáxima) e o momento (pré versus pós-exercício) como os fatores principais. Quando os valores pré-exercício foram significativamente diferentes entre as sessões (para FC e DP), empregou-se uma análise de covariância (ANCOVA) usando o valor pré-exercício como covariável. Foi utilizado o teste post-hoc de Newman-Keuls para identificar as significâncias quando apropriado. Foi considerado significativo $p<0,05$ e os dados foram apresentados como média \pm desvio padrão (DP) para variáveis contínuas e como frequência de aparecimento (\%) para variáveis categóricas, como comorbidades e uso de medicamentos.

\section{Resultados}

Inicialmente, 50 pacientes se ofereceram como voluntários para o estudo, 11 dos quais se abstiveram de participar devido à falta de tempo. Deste modo, 39 pacientes assinaram o termo de consentimento livre e esclarecido e realizaram os exames preliminares, dos quais 9 foram excluídos (5 devido a anormalidades de ECG no teste ergométrico e 4 devido à interrupção do teste ergométrico por outros motivos que não a dor claudicante). Portanto, 30 pacientes realizaram as sessões experimentais máximas e submáximas e suas características são apresentadas na Tabela 1.

As respostas hemodinâmicas e autonômicas são apresentadas na Tabela 2. A PA sistólica e média diminuíram após a sessão submáxima e aumentaram após a sessão máxima (interações, $p<0,001$ para ambas). A PA diastólica aumentou apenas após a caminhada máxima (interação, $p<$ 0,001). A FC e o DP pré-exercício foram significativamente maiores na sessão submáxima de caminhada do que na máxima, e a ANCOVA revelou que estas diferenças préexercício não afetaram os resultados. Assim, a FC apresentou aumentos semelhantes após os períodos de caminhada máxima e submáxima (momento, $p<0,001$ ), enquanto o DP aumentou significativamente apenas após a caminhada máxima (interação, $\mathrm{p}=0,007$ ).

A FA diminuiu enquanto a $F B$ e a relação $F B / F A$ aumentaram significativamente e de forma semelhante após as sessões de caminhada máxima e submáxima (momento, $p$ $=0,02, p=0,05$ e $p=0,04$, respectivamente). 


\section{Tabela 1 - Características dos pacientes}

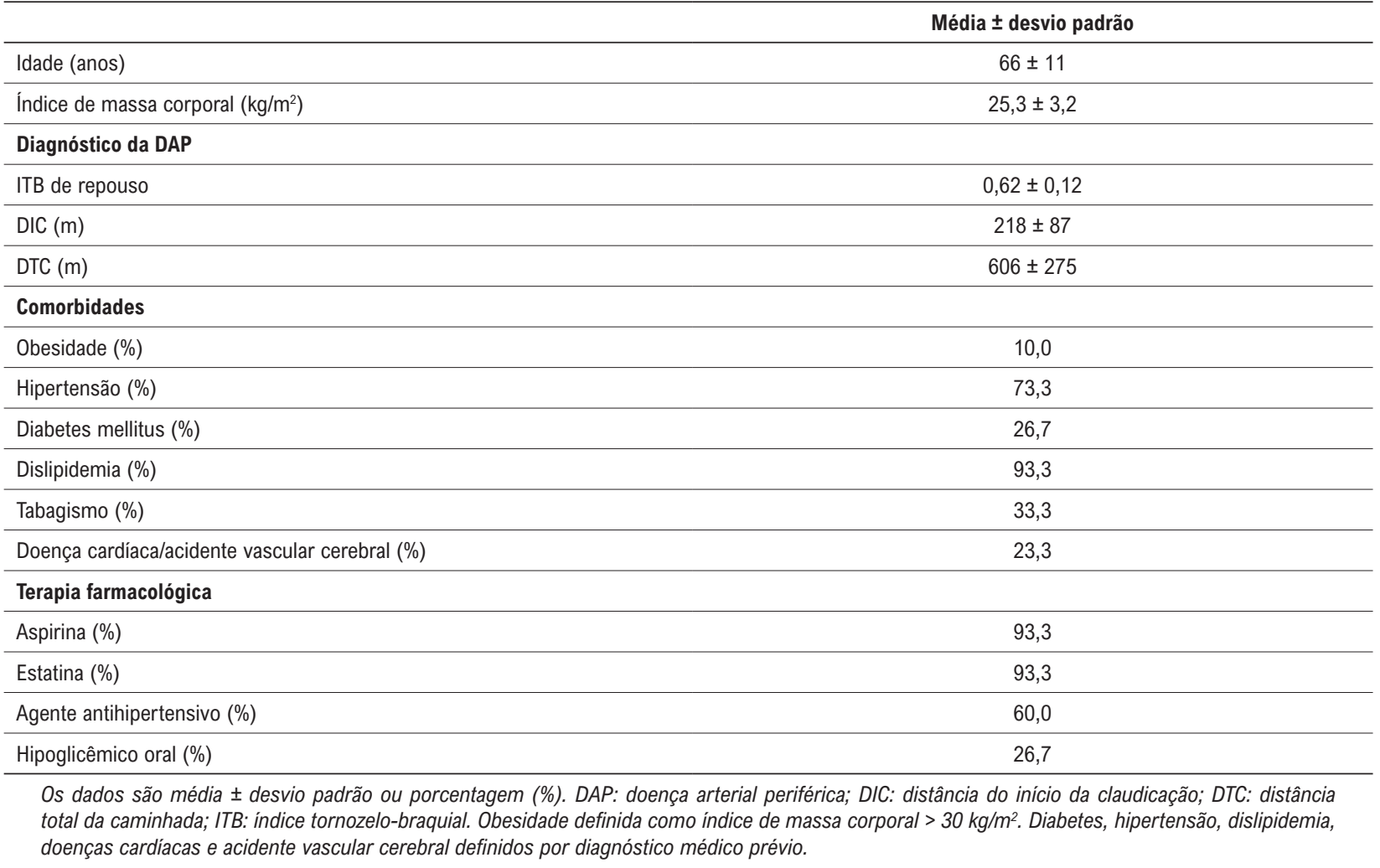

Tabela 2 - Variáveis hemodinâmicas e autonômicas medidas pré e pós-exercício nas sessões de caminhada submáxima e máxima

\begin{tabular}{|c|c|c|c|c|c|c|c|}
\hline & \multicolumn{2}{|c|}{ Submáxima } & \multicolumn{2}{|c|}{ Máxima } & \multirow[b]{2}{*}{ p sessão } & \multirow[b]{2}{*}{ p momento } & \multirow[b]{2}{*}{ p interação } \\
\hline & Pré & Pós & Pré & Pós & & & \\
\hline \multicolumn{8}{|c|}{ Hemodinâmica sistêmica $(\mathrm{N}=30)$} \\
\hline PA sistólica (mmHg) & $132 \pm 16$ & $125 \pm 15^{\star} \#$ & $134 \pm 13$ & $138 \pm 17^{*}$ & 0,01 & 0,18 & 0,01 \\
\hline PA diastólica (mmHg) & $77 \pm 9$ & $76 \pm 8 \#$ & $78 \pm 8$ & $83 \pm 9^{*}$ & 0,01 & 0,01 & 0,01 \\
\hline PA média $(\mathrm{mmHg})$ & $95 \pm 10$ & $92 \pm 9^{*} \#$ & $96 \pm 9$ & $101 \pm 10^{*}$ & 0,01 & 0,05 & 0,01 \\
\hline $\mathrm{FC}(\mathrm{bpm})$ & $64 \pm 9 \#$ & $67 \pm 9^{*} \#$ & $68 \pm 9$ & $71 \pm 10^{*}$ & 0,01 & 0,01 & 0,70 \\
\hline $\mathrm{DP}\left(\mathrm{bpm}^{*} \mathrm{mmHg}\right)$ & $8466 \pm 1466 \#$ & $8308 \pm 1433 \#$ & $9010 \pm 1394$ & $9762 \pm 1671^{*}$ & 0,01 & 0,01 & 0,01 \\
\hline \multicolumn{8}{|c|}{ Modulação autonômica (n=22) } \\
\hline FB (un) & $56 \pm 22$ & $64 \pm 20^{*}$ & $51 \pm 18$ & $60 \pm 21^{*}$ & 0,12 & 0,05 & 0,75 \\
\hline FA (un) & $40 \pm 21$ & $31 \pm 19^{*}$ & $44 \pm 17$ & $34 \pm 19^{*}$ & 0,19 & 0,02 & 0,88 \\
\hline Relação FB/FA & $0,2 \pm 0,5$ & $0,4 \pm 0,4^{*}$ & $0,1 \pm 0,4$ & $0,3 \pm 0,5^{*}$ & 0,11 & 0,04 & 0,74 \\
\hline \multicolumn{8}{|c|}{ Hemodinâmica local $(n=21)$} \\
\hline FS do antebraço & $1,42 \pm 0,63$ & $1,68 \pm 0,68^{*}$ & $1,41 \pm 0,59$ & $1,65 \pm 0,67^{*}$ & 0,70 & 0,01 & 0,59 \\
\hline FS da panturrilha & $12,0 \pm 7,1$ & $13,7 \pm 7,0^{*}$ & $12,2 \pm 7,8$ & $14,4 \pm 8,8^{*}$ & 0,11 & 0,01 & 0,57 \\
\hline RV do antebraço & $80,9 \pm 34,8$ & $67,2 \pm 30,0^{*}$ & $83,9 \pm 40,6$ & $73,6 \pm 34,1^{*}$ & 0,18 & 0,01 & 0,14 \\
\hline RV da panturrilha & $56,8 \pm 30,2$ & $40,4 \pm 19,8^{*}$ & $63,9 \pm 29,5$ & $52,4 \pm 26,5^{*}$ & 0,02 & 0,01 & 0,52 \\
\hline AUCFS do antebraço & $1085 \pm 507$ & $1299 \pm 609$ & $1294 \pm 676$ & $1218 \pm 476$ & 0,66 & 0,38 & 0,50 \\
\hline AUCFS da panturrilha & $1081 \pm 606$ & $1152 \pm 603$ & $999 \pm 467$ & $1270 \pm 825$ & 0,89 & 0,13 & 0,20 \\
\hline
\end{tabular}

Os dados são média \pm desvio padrão. AUC: área sob a curva; DP: duplo-produto; FA: frequência alta; FB: frequência baixa; FC: frequência cardíaca; FS: fluxo sanguíneo; PA: pressão arterial; RV: resistência vascular; un: unidades normalizadas. Valores para FS são ml. $100 \mathrm{ml}$ tecido-1. min ${ }^{-1} .{ }^{*}=$ diferente do $^{2}$ pré na mesma sessão $(p<0,05)$; \# = diferente da sessão máxima no mesmo momento $(p<0,05)$. Análises realizadas por ANOVA bilateral. 
Os FS do antebraço e da panturrilha aumentaram significativamente e de forma semelhante após as sessões de caminhada máxima e submáxima (momento, p < 0,001), enquanto a resistência vascular do antebraço e da panturrilha diminuiu de forma semelhante após ambos os períodos de caminhada (momento, $p<0,001$ e $p=0,01$, respectivamente), e a AUCFS do antebraço e da panturrilha não se alterou após os períodos de caminhada submáxima ou máxima (todos $p>0,05$ ).

As respostas sanguíneas são mostradas na Tabela 3. ON, peroxidação lipídica, CRP e TNF- $\alpha$ não se alteraram após as sessões de caminhada submáxima ou máxima (todos $p$ $>0,05)$, enquanto ICAM e VCAM exibiram um aumento semelhante e significativo após as sessões de caminhada máxima e submáxima (momento, $\mathrm{p}=0,001$ para ambos).

\section{Discussão}

Os achados principais do presente estudo foram que os pacientes com a DAP sintomática apresentaram: 1) redução da PA sistólica após a caminhada submáxima, bem como aumento da PA sistólica após a caminhada máxima; 2) aumento do DP apenas após a caminhada máxima; 3) aumentos semelhantes nos níveis de FC, relação FB/FA, FB, ICAM e VCAM após as sessões de caminhada máxima e submáxima; e 4) nenhuma alteração no $\mathrm{ON}$ ou na capacidade vasodilatadora após as sessões de caminhada máxima ou submáxima.

Realizar caminhada até a dor submáxima, mas não máxima, diminuiu a PA pós-exercício. Estudos anteriores ${ }^{17,24}$ já relataram a ocorrência da hipotensão pós-exercício (HPE, ou seja, uma diminuição da PA após uma sessão de exercício em comparação com os valores pré-exercício ${ }^{25,26}$ em pacientes com DAP sintomática após realizar caminhada até dor moderada. A novidade deste estudo foi fornecer evidências de que, em pacientes com DAP no estágio II de Fontaine, a HPE não ocorreu quando a caminhada foi realizada até a dor máxima e a PA permaneceu elevada após a caminhada máxima. Visto que a HPE é conhecida como um fenômeno clinicamente relevante em populações hipertensas, ${ }^{27}$ a caminhada submáxima, mas não máxima, pode produzir benefícios hipotensivos agudos em pacientes com $\mathrm{Cl}$ e hipertensão. Além disso, evidências recentes têm mostrado que a HPE se correlaciona com diminuições na PA após um período de treinamento e é um possível preditor da responsividade crônica. ${ }^{28,29}$ Desta maneira, estes resultados levantam a hipótese de que a caminhada submáxima pode produzir melhores efeitos hipotensivos crônicos do que a caminhada máxima nesta população. Isto precisa ser testado em estudos futuros.

A FC pós-exercício aumentou de forma semelhante após as sessões de caminhada máxima e submáxima, o que é consistente com o aumento semelhante observado nas alterações da modulação autonômica cardíaca em direção à predominância simpática após ambas as sessões de caminhada (ou seja, um aumento semelhante na $\mathrm{FB}$ e na relação $\mathrm{FB} /$ FA, bem como uma diminuição na FA). ${ }^{30}$ Esta ausência de diferença entre as sessões máximas e submáximas foi, em certa medida, inesperada, visto que, em outras populações, as alterações na FC pós-exercício e na modulação simpatovagal costumam estar associadas à intensidade do exercício. ${ }^{31}$ Este resultado aparentemente contraditório pode ser explicado pelo fato de a sessão de caminhada submáxima ter durado mais (30 minutos, distância total percorrida $=1600 \mathrm{~m}$ ) do que a sessão máxima (12 \pm 5 minutos, distância total percorrida $=606 \pm 275 \mathrm{~m}$ ). Assim, como a dor produz ativação simpática, ${ }^{32}$ é possível que, apesar da intensidade moderada, o maior período de dor na sessão submáxima possa ter levado a um aumento sustentado da modulação simpática e, consequentemente, da FC durante o período de recuperação, igualando-se ao aumento produzido pela sessão máxima mais intensa, mas mais curta. Adicionalmente, embora a FC pós-exercício tenha aumentado em ambas as sessões de caminhada, a PA diminuiu apenas na sessão de caminhada submáxima, consequentemente levando a um DP mais elevado após a caminhada máxima, o que reflete maior sobrecarga cardíaca e, consequentemente, maior risco de eventos adversos agudos após a caminhada máxima. ${ }^{14}$ Portanto, estes resultados sugerem que a caminhada

Tabela 3 - Concentrações plasmáticas de óxido nítrico, estresse oxidativo e variáveis inflamatórias medidas pré e pós-exercício nas sessões de caminhada submáxima e máxima

\begin{tabular}{|c|c|c|c|c|c|c|c|}
\hline & \multicolumn{2}{|c|}{ Submáxima } & \multicolumn{2}{|c|}{ Máxima } & \multirow[b]{2}{*}{ p sessão } & \multirow[b]{2}{*}{ p momento } & \multirow[b]{2}{*}{ p interação } \\
\hline & Pré & Pós & Pré & Pós & & & \\
\hline ON $(\mu \mathrm{M})$ & $14,32 \pm 5,65$ & $13,59 \pm 4,63$ & $13,53 \pm 4,51$ & $13,68 \pm 4,21$ & 0,29 & 0,24 & 0,57 \\
\hline \multicolumn{8}{|c|}{ Estresse oxidativo } \\
\hline $\mathrm{PL}(\mu \mathrm{M})$ & $18,81 \pm 14,69$ & $19,29 \pm 15,34$ & $18,71 \pm 17,06$ & $20,55 \pm 19,01$ & 0,81 & 0,44 & 0,77 \\
\hline \multicolumn{8}{|l|}{ Inflamação } \\
\hline CRP (pg/ml) & $1868 \pm 1435$ & $1843 \pm 1485$ & $1614 \pm 1651$ & $1837 \pm 1586$ & 0,41 & 0,13 & 0,45 \\
\hline TNF- $\alpha(p g / m l)$ & $1,18 \pm 0,36$ & $1,24 \pm 0,29$ & $1,21 \pm 0,28$ & $1,23 \pm 0,25$ & 0,75 & 0,21 & 0,57 \\
\hline ICAM (ng/ml) & $223 \pm 96$ & $236 \pm 99^{*}$ & $218 \pm 92$ & $244 \pm 100^{*}$ & 0,74 & 0,01 & 0,08 \\
\hline VCAM (ng/ml) & $619 \pm 250$ & $671 \pm 286^{*}$ & $592 \pm 237$ & $650 \pm 247^{*}$ & 0,16 & 0,01 & 0,75 \\
\hline
\end{tabular}

Os dados são média \pm desvio padrão. CRP: proteína C-reativa; ICAM: molécula de adesão intercelular; ON: óxido nitrico; PL: peroxidação lipídica; TNF- $\alpha$ : fator de necrose tumoral-a; VCAM: molécula de adesão de células vasculares. ${ }^{*}=$ diferente do pré na mesma sessão ( $\left.p<0,05\right)$. Análises realizadas por ANOVA bilateral. 
submáxima pode ser mais segura para pacientes predispostos a eventos cardiovasculares agudos.

Os FS do antebraço e da panturrilha aumentaram de forma semelhante após as sessões de caminhada submáxima e máxima, e essas respostas estão de acordo com estudos anteriores. ${ }^{17,33}$ No entanto, é de interesse notar que a capacidade vasodilatadora não alterou após qualquer uma das duas sessões de caminhada, embora estudos anteriores tenham relatado uma diminuição da função endotelial após caminhada máxima. ${ }^{12,34}$ Possíveis diferenças entre os estudos podem estar relacionadas aos métodos usados para avaliar a função vascular (pletismógrafo versus ultrassom). No entanto, no presente estudo, a ausência de alterações na capacidade vasodilatadora está de acordo com a manutenção dos marcadores do $\mathrm{ON}$ e do estresse oxidativo.

Conforme o esperado, as sessões de caminhada máxima e submáxima aumentaram os marcadores inflamatórios. Porém, diferentemente da hipótese, a inflamação aumentou de forma semelhante após as duas sessões. Mais uma vez, essa resposta pode estar relacionada ao fato de que a duração do exercício foi maior na sessão de caminhada submáxima, levando a uma magnitude semelhante de inflamação, apesar do menor grau da dor.

A ausência de volume pareado entre as duas sessões de caminhada é uma limitação deste estudo, o que nos impede de atribuir os resultados apenas ao grau de dor. No entanto, como um estudo inicial a comparar as respostas máximas e submáximas pós-exercício, o presente estudo optou pela utilização de um protocolo máximo amplamente investigado na literatura ${ }^{7,11,34}$ e um protocolo submáximo, ambos os quais já demonstraram produzir benefícios cardiovasculares. ${ }^{8,17}$ Estudos futuros devem comparar outros protocolos máximos e submáximos com volume semelhante. Além disso, é importante mencionar que este estudo foi realizado com homens nos estágios Ila e IIb de Fountain, e as respostas póscaminhada podem diferir em mulheres, em pacientes em outros estágios da doença e em pacientes com características clínicas diferentes, apesar de estágio II de Fontaine. Estudos futuros poderão superar essas limitações estudando mulheres e outros pacientes com DAP. Além disso, as medidas foram realizadas em apenas um momento durante o período pósexercício. Para melhor compreensão das respostas, deve ser realizado um acompanhamento durante um período mais longo, com mais medidas, em investigações futuras.

\section{Conclusões}

Em pacientes do sexo masculino com a DAP sintomática, caminhar até a dor submáxima, mas não máxima, reduziu a PA pós-exercício, enquanto apenas a caminhada máxima elevou o DP pós-exercício. Por outro lado, as sessões de caminhada máxima e submáxima produziram aumentos pós-exercício semelhantes na FC, equilíbrio simpatovagal cardíaco, FS e inflamação.

\section{Implicações práticas}

- Caminhada submáxima, mas não máxima, reduz a PA no período pós-exercício.

- Apenas a caminhada máxima aumenta a carga cardíaca pós-exercício.

- Caminhada submáxima e máxima aumentam a inflamação pós-exercício de modo semelhante.

- A caminhada submáxima pode ser mais adequada do que a caminhada máxima para pacientes com DAP sintomática, porque resulta em menor risco cardiovascular agudo durante o período de recuperação.

\section{Contribuição dos autores}

Concepção e desenho da pesquisa: Chehuen M, AndradeLima A, Silva Junior N, Leicht A, Brum PC, Oliveira EM, Wolosker N, Forjaz CLM; Obtenção de dados: Chehuen $M$, Andrade-Lima A, Silva Junior N, Miyasato R, Souza R; Análise e interpretação dos dados: Chehuen M, Andrade-Lima A, Silva Junior N, Miyasato R, Souza R, Leicht A, Brum PC, Oliveira EM, Wolosker N, Forjaz CLM; Análise estatística: Chehuen M, Andrade-Lima A, Forjaz CLM; Obtenção de financiamento: Forjaz CLM; Redação do manuscrito: Chehuen M, AndradeLima A, Leicht A, Brum PC, Oliveira EM, Wolosker N, Forjaz CLM; Revisão crítica do manuscrito quanto ao conteúdo intelectual importante: Chehuen M, Andrade-Lima A, Silva Junior N, Souza R, Leicht A, Brum PC, Oliveira EM, Wolosker $\mathrm{N}$, Forjaz CLM.

\section{Potencial conflito de interesse}

Não há conflito com o presente artigo

\section{Fontes de financiamento}

O presente estudo não teve fontes de financiamento externas.

\section{Vinculação acadêmica}

Este artigo é parte de tese de pós-doutorado de Marcel Chehuen pela Universidade de São Paulo.

\section{Referências}

1. Hirsch AT, Haskal ZJ, Hertzer NR, Bakal CW, Creager MA, Halperin JL, et al. ACC/AHA 2005 Practice Guidelines for the management of patients with peripheral arterial disease (lower extremity, renal, mesenteric, and abdominal aortic): a collaborative report from the American Association for Vascular Surgery/Society for Vascular Surgery, Society for Cardiovascular Angiography and Interventions, Society for Vascular Medicine and Biology, Society of

Interventional Radiology, and the ACC/AHA Task Force on Practice Guidelines (Writing Committee to Develop Guidelines for the Management of Patients With Peripheral Arterial Disease): endorsed by the American Association of Cardiovascular and Pulmonary Rehabilitation; National Heart, Lung, and Blood Institute; Society for Vascular Nursing; TransAtlantic Inter-Society Consensus; and Vascular Disease Foundation. Circulation. 2006;113(11):e463-654. 
2. Norgren L, Hiatt WR, Dormandy JA, Nehler MR, Harris KA, Fowkes FG, et al. Inter-society consensus for the management of peripheral arterial disease. Int Angiol. 2007;26(2):81-157

3. Bhatt DL, Steg PG, Ohman EM, Hirsch AT, Ikeda Y, Mas JL, et al. International prevalence, recognition, and treatment of cardiovascular risk factors in outpatients with atherothrombosis. JAMA. 2006;295(2):180-9.

4. Goernig M, Schroeder R, Roth T, Truebner S, Palutke I, Figulla HR, et al. Peripheral arterial disease alters heart rate variability in cardiovascular patients. Pacing Clin Electrophysiol. 2008;31(7):858-62.

5. Brevetti G, Giugliano G, Brevetti L, Hiatt WR. Inflammation in doença arterial periférica. Circulation. 2010;122(18):1862-75.

6. Chapman MJ. From pathophysiology to targeted therapy for atherothrombosis: a role for the combination of statin and aspirin in secondary prevention. Pharmacol Ther. 2007;113(1):184-96.

7. Signorelli SS, Mazzarino MC, Di Pino L, Malaponte G, Porto C, Pennisi G, et al. High circulating levels of cytokines (IL-6 and TNFalpha), adhesion molecules (VCAM-1 and ICAM- 1 ) and selectins in patients with peripheral arterial disease at rest and after a treadmill test. Vasc Med. 2003;8(1):15-9.

8. Chehuen M, Cucato GG, Carvalho CRF, Ritti-Dias RM, Wolosker N, Leicht AS, et al. Walking training at the heart rate of pain threshold improves cardiovascular function and autonomic regulation in intermittent claudication: A randomized controlled trial. J Sci Med Sport. 2017;20(10):886-92.

9. Gerhard-Herman MD, Gornik HL, Barrett C, Barshes NR, Corriere MA, Drachman DE, et al. 2016 AHA/ACC Guideline on the Management of Patients With Lower Extremity Doença arterial periférica: Executive Summary: A Report of the American College of Cardiology/American Heart Association Task Force on Clinical Practice Guidelines. Circulation. 2017;135(12):e686-e725

10. Luttrell MJ, Halliwill JR. Recovery from exercise: vulnerable state, window of opportunity, or crystal ball? Front Physiol. 2015;6:204.

11. Palmer-Kazen $U$, Religa $P, W a h l b e r g ~ E$. Exercise in patients with intermittent claudication elicits signs of inflammation and angiogenesis. Eur J Vasc Endovasc Surg. 2009;38(6):689-96.

12. Silvestro A, Scopacasa F, Oliva G, de Cristofaro T, Iuliano L, Brevetti G. Vitamin C prevents endothelial dysfunction induced by acute exercise in patients with intermittent claudication. Atherosclerosis. $2002 ; 165(2): 277-83$

13. Ritti-Dias RM, Meneses AL, Parker DE, Montgomery PS, Khurana A, Gardne AW. Cardiovascular responses to walking in patients with doença arterial periférica. Med Sci Sports Exerc. 2011;43(11):2017-23.

14. Svensson P, Niklasson U, Ostergren J. Episodes of ST-segment depression is related to changes in ambulatory blood pressure and heart rate in intermittent claudication. J Intern Med. 2001;250(5):398-405.

15. Novakovic M, Krevel B, Rajkovic U, Vizintin Cuderman T, Jansa Trontelj K, Fras Z, et al. Moderate-pain versus pain-free exercise, walking capacity, and cardiovascular health in patients with doença arterial periférica. J Vasc Surg. 2019;70(1):148-56

16. Cucato GG, Chehuen Mda R, Costa LA, Ritti-Dias RM, Wolosker N, Saxton $J M$, et al. Exercise prescription using the heart of claudication pain onset in patients with intermittent claudication. Clinics (Sao Paulo). 2013;68(7):974-8.

17. Cucato GG, Chehuen Mda R, Ritti-Dias RM, Carvalho CR, Wolosker $\mathrm{N}$, Saxton JM, et al. Post-walking exercise hypotension in patients with intermittent claudication. Med Sci Sports Exerc. 2015;47(3):460-7.

18. Gardner AW, Skinner JS, Cantwell BW, Smith LK. Progressive vs singlestage treadmill tests for evaluation of claudication. Med Sci Sports Exerc. $1991 ; 23(4): 402-8$
19. Hall WL, Vafeiadou K, Hallund J, Bugel S, Koebnick C, Reimann M, et al. Soyisoflavone-enriched foods and inflammatory biomarkers of cardiovascula disease risk in postmenopausal women: interactions with genotype and equol production. Am J Clin Nutr. 2005;82(6):1260-8; quiz 365-6.

20. Smith TJ, Karl JP, Wilson MA, Whitney CC, Barrett A, Farhadi NF, et al. Glycaemic regulation, appetite and ex vivo oxidative stress in young adults following consumption of high-carbohydrate cereal bars fortified with polyphenol-rich berries. Br J Nutr. 2019;121(9):1026-38.

21. White WB. Heart rate and the rate-pressure product as determinants of cardiovascular risk in patients with hypertension. Am J Hypertens. 1999;12(2 Pt 2):50S-5S

22. Heart rate variability. Standards of measurement, physiological interpretation, and clinical use. Task Force of the European Society of Cardiology and the North American Society of Pacing and Electrophysiology. Eur Heart J. 1996;17(3):354-81.

23. Thijssen DH, Bleeker MW, Smits P, Hopman MT. Reproducibility of blood flow and post-occlusive reactive hyperaemia as measured by venous occlusion plethysmography. Clin Sci (Lond). 2005;108(2):151-7.

24. Cavalcante BR, Ritti-Dias RM, Soares AH, Lima AH, Correia MA, De Matos LD, et al. A Single Bout of Arm-crank Exercise Promotes Positive Emotions and Post-Exercise Hypotension in Patients with Symptomatic Doença arterial periférica. Eur J Vasc Endovasc Surg. 2017;53(2):223-8.

25. de Brito LC, Fecchio RY, Pecanha T, Lima A, Halliwill J, Forjaz CLM. Recommendations in Post-exercise Hypotension: Concerns, Best Practices and Interpretation. Int J Sports Med. 2019;40(8):487-97.

26. Kenney MJ, Seals DR. Postexercise hypotension. Key features, mechanisms, and clinical significance. Hypertension. 1993;22(5):653-64.

27. Pescatello LS, Franklin BA, Fagard R, Farquhar WB, Kelley GA, Ray CA et al. American College of Sports Medicine position stand. Exercise and hypertension. Med Sci Sports Exerc. 2004;36(3):533-53.

28. Kiviniemi AM, Hautala AJ, Karjalainen JJ, Piira OP, Lepojarvi S, Ukkola O et al. Acute post-exercise change in blood pressure and exercise training response in patients with coronary artery disease. Front Physiol. 2014;5:526.

29. Moreira SR, Cucato GG, Terra DF, Ritti-Dias RM. Acute blood pressure changes are related to chronic effects of resistance exercise in medicated hypertensives elderly women. Clin Physiol Funct Imaging. 2016;36(3):242-8.

30. de Brito LC, Rezende RA, da Silva Junior ND, Tinucci T, Casarini DE, CipollaNeto J, et al. Post-Exercise Hypotension and Its Mechanisms Differ after Morning and Evening Exercise: A Randomized Crossover Study. PLoS One. 2015;10(7):e0132458.

31. Cote AT, Bredin SS, Phillips AA, Koehle MS, Warburton DE. Greate autonomic modulation during post-exercise hypotension following highintensity interval exercise in endurance-trained men and women. Eur J Appl Physiol. 2015;115(1):81-9.

32. Schlereth T, Birklein F. The sympathetic nervous system and pain. Neuromolecular Med. 2008;10(3):141-7.

33. da Silva ND, Jr., Roseguini BT, Chehuen M, Fernandes T, Mota GF, Martin PK, et al. Effects of oral $\mathrm{N}$-acetylcysteine on walking capacity, leg reactive hyperemia, and inflammatory and angiogenic mediators in patients with intermittent claudication. Am J Physiol Heart Circ Physiol. 2015;309(5):H897-905.

34. Allen JD, Stabler T, Kenjale A, Ham KL, Robbins JL, Duscha BD, et al. Plasma nitrite flux predicts exercise performance in peripheral arterial disease after 3months of exercise training. Free Radic Biol Med. 2010;49(6):1138-44. 